\title{
Conservation Laws for a Degasperis Procesi Equation and a Coupled Variable-Coefficient Modified Korteweg-de Vries System in a Two-Layer Fluid Model via the Multiplier Approach
}

\author{
E. Osman, ${ }^{1}$ M. Khalfallah, ${ }^{2}$ and H. Sapoor ${ }^{1}$ \\ ${ }^{1}$ Department of Mathematics, Faculty of Science, Sohag University, Sohag 82524, Egypt \\ ${ }^{2}$ Mathematics Department, Faculty of Science, South Valley University, Qena, Egypt \\ Correspondence should be addressed to H. Sapoor; hussien.abdalnaeem1@science.sohag.edu.eg
}

Received 21 May 2014; Revised 20 September 2014; Accepted 16 October 2014; Published 13 November 2014

Academic Editor: Athanasios N. Yannacopoulos

Copyright (C) 2014 E. Osman et al. This is an open access article distributed under the Creative Commons Attribution License, which permits unrestricted use, distribution, and reproduction in any medium, provided the original work is properly cited.

We employ the multiplier approach (variational derivative method) to derive the conservation laws for the Degasperis Procesi equation and a coupled variable-coefficient modified Korteweg-de Vries system in a two-layer fluid model. Firstly, the multipliers are computed and then conserved vectors are obtained for each multiplier.

\section{Introduction}

The conservation laws are important in the solution and reductions of partial differential equations. Conservation law, also called law of conservation, in physics, is several principles that state that certain physical properties (i.e., measurable quantities) do not change in the course of time within an isolated physical system. In classical physics, laws of this type govern energy, momentum, angular momentum, mass, and electric charge. In particle physics, other conservation laws apply to properties of subatomic particles that are invariant during interactions. An important function of conservation laws is that they make it possible to predict the macroscopic behaviour of a system without having to consider the microscopic details of the course of a physical process or chemical reaction. Many powerful methods have been developed for the construction of conservation laws, such as The Laplace Direct method [1], multiplier approach $[2,3]$, Kara and Mahomed symmetry condition [4], Wolf [5, 6], Göktaş and Hereman [7], Hereman et al. [8-10], and Cheviakov [11] who developed powerful software packages to compute conservation laws for partial differential equations. Infinitely many conservation laws are obtained based on the Lax pair via the Hirota method and symbolic computation, bilinear forms, bilinear Backlund transformations, and one- and two-soliton-like solutions are also derived. With different coefficients, bell-shaped, periodic-changing, quadratic-varying, exponential-decreasing, and exponentialincreasing soliton-like profiles are obtained in [12]. Also by the spectral analysis the Hamiltonian and periodicity of the qZK equation are investigated by usig the Hirota method [13]. The nonautonomous matter waves with timedependent modulation in a one-dimensional trapped spin-1 Bose-Einstein condensate and the generalized three-coupled Gross-Pitaevskii equations by means of the Hirota bilinear method are studied in [14]. The multiplier approach (also known as variational derivative method) was proposed by Steudel [15] who wrote the conservation law in characteristic form as $D_{i} T^{i}=A^{\alpha} E_{\alpha}$. Later, Olver [16] modified the method of determining the characteristics (multipliers) by taking the variational derivative of $D_{i} T^{i}=Q^{\alpha} E_{\alpha}$ not only for the arbitrary functions, but also for solutions of system of partial differential equations. The outline of the paper is as follows. In Section 2, some definitions related to the multiplier approach are given. In Section 3, conservation laws for the Degasperis Procesi equation are derived by first computing the multipliers. The conservation laws for a coupled variable-coefficient modified Korteweg-de Vries system in a two-layer fluid model are derived in Section 4. Finally, conclusions are summarized in Section 5. 


\section{Necessary Preliminaries}

Let $x^{i}, i=1,2, \ldots, n$, be $n$ independent variables and let $u$ be the dependent variable.

(1) The total derivative operator with respect to $x^{i}$ is

$D_{i}=\frac{\partial}{\partial x^{i}}+u_{i} \frac{\partial}{\partial u}+u_{i j} \frac{\partial}{\partial u_{j}}+\cdots \quad i=1,2, \ldots, n$,

where $u_{i}$ denotes the derivative of $u$ with respect to $x^{i}$. Similarly $u_{i j}$ denotes the derivative of $u$ with respect to $x^{i}$ and $x^{j}$.

(2) The Euler operator is defined by

$\frac{\delta}{\delta u}=\frac{\partial}{\partial u}-D_{i} \frac{\partial}{\partial u_{i}}+D_{i j} \frac{\partial}{\partial u_{i j}}-D_{i j k} \frac{\partial}{\partial u_{i j k}}+\cdots$.

Consider a $k$ th-order partial differential equation of $n$ independent and one dependent variable

$$
E\left(x, u, u_{(1)}, u_{(2)}, \ldots, u_{(k)}\right)=0 .
$$

(3) An $n$-tuple $T=\left(T^{1}, T^{2}, \ldots, T^{n}\right), i=1,2, \ldots, n$, such that

$$
D_{i} T^{i}=0
$$

holds for all solutions of (3) is known as the conserved vector of (3).

(4) The multiplier $A$ of system (3) has the property

$$
D_{i} T^{i}=A E
$$

for arbitrary function $u\left(x^{1}, x^{2}, \ldots, x^{n}\right)[16]$.

(5) The determining equations for multipliers are obtained by taking the variational derivative of (5) (see [16]):

$$
\frac{\delta}{\delta u}(A E)=0
$$

Equation (6) holds for arbitrary function $u\left(x^{1}, x^{2}, \ldots\right.$, $\left.x^{n}\right)$ not only for solutions of system (3).

Once the multipliers are computed from (6), the conserved vectors can be derived systematically using (5) as the determining equation. But in some problems it is not difficult to construct the conserved vectors by elementary manipulations after the determination of the multipliers.

\section{Conservation Laws for the Degasperis Procesi Equation}

The Degasperis Procesi equation [17] takes the form

$$
u_{t}-u_{x x t}+4 u u_{x}-3 u_{x} u_{x x}-u u_{x x x}=0 .
$$

The Degasperis Procesi equation (7) is very interesting as it is an integrable shallow water equation and presents a quite rich structure. Also it can be used to model wave perturbations in relaxing media.

We will derive the conservation laws for (7) by the multiplier approach. The determining equation for multiplier $A(t$, $x, u)$, from $(6)$, is

$$
\frac{\delta}{\delta u}\left[A\left(u_{t}-u_{x x t}+4 u u_{x}-3 u_{x} u_{x x}-u u_{x x x}\right)\right]=0 .
$$

The standard Euler operator $\delta / \delta u$ from (2) can be defined as

$$
\begin{gathered}
\frac{\delta}{\delta u}=\frac{\partial}{\partial u}-D_{t} \frac{\partial}{\partial u_{t}}-D_{x} \frac{\partial}{\partial u_{x}}+D_{t}^{2} \frac{\partial}{\partial u_{t t}} \\
+D_{x}^{2} \frac{\partial}{\partial u_{x x}}+D_{x} D_{t} \frac{\partial}{\partial u_{t x}}-\cdots,
\end{gathered}
$$

and total derivative operators $D_{t}$ and $D_{x}$ using (1) are

$$
\begin{aligned}
D_{t} & =\frac{\partial}{\partial t}+u_{t} \frac{\partial}{\partial u}+u_{t t} \frac{\partial}{\partial u_{t}}+u_{t x} \frac{\partial}{\partial u_{x}}+\cdots \\
D_{x} & =\frac{\partial}{\partial t}+u_{x} \frac{\partial}{\partial u}+u_{x x} \frac{\partial}{\partial u_{x}}+u_{t x} \frac{\partial}{\partial u_{t}}+\cdots
\end{aligned}
$$

Equation (8) after expansion and simplification takes the following form:

$$
\begin{aligned}
u_{x x} & \left(A_{u t}+3 u A_{x u}+3 u u_{x} A_{u u}-3 u_{x} A_{u}+u_{t} A_{u u}\right) \\
& +u_{x}^{2}\left(u A_{u u x}+A_{u u t}+u_{t} A_{u u u}\right)+u u_{x}^{3} A_{u u u} \\
& +u_{x t}\left(2 A_{x u}+2 u_{x} A_{u u}\right)+u_{x}\left(2 A_{x t u}+3 u A_{x x u}\right) \\
& +u_{t}\left(A_{x x u}+2 u_{x} A_{x u u}\right)+u\left(A_{x x x}-4 A_{x}\right) \\
& +A_{x x t}-A_{t}=0
\end{aligned}
$$

which yields

$$
A=c_{1}+c_{2} e^{-2 x}+c_{3} e^{2 x}
$$

From (5) and (12), we have

$$
\begin{aligned}
& \left(c_{1}+c_{2} e^{-2 x}+c_{3} e^{2 x}\right) \\
& \times\left(u_{t}-u_{x x t}+4 u u_{x}-3 u_{x} u_{x x}-u u_{x x x}\right) \\
& =D_{t}\left[c_{1}\left(u-u_{x x}\right)+c_{2}\left(u e^{-2 x}-u_{x x} e^{-2 x}\right)\right. \\
& \left.+c_{3}\left(u e^{2 x}-u_{x x} e^{2 x}\right)\right] \\
& +D_{x}\left[c_{1}\left(2 u^{2}-u_{x}^{2}-u u_{x x}\right)\right. \\
& \quad+c_{2}\left(-2 u u_{x} e^{-2 x}-u_{x}^{2} e^{-2 x}-u u_{x x} e^{-2 x}\right) \\
& \left.\quad+c_{3}\left(2 u u_{x} e^{2 x}-u_{x}^{2} e^{2 x}-u u_{x x} e^{2 x}\right)\right]
\end{aligned}
$$


for arbitrary functions $u(t, x)$. When $u(t, x)$ is solutions of $(7)$ then left hand side of (13) vanishes and we obtain

$$
\begin{aligned}
D_{t}\left[c_{1}\left(u-u_{x x}\right)+c_{2}\left(u e^{-2 x}-u_{x x} e^{-2 x}\right)\right. & \\
+ & \left.c_{3}\left(u e^{2 x}-u_{x x} e^{2 x}\right)\right] \\
+D_{x}[ & c_{1}\left(2 u^{2}-u_{x}^{2}-u u_{x x}\right) \\
& +c_{2}\left(-2 u u_{x} e^{-2 x}-u_{x}^{2} e^{-2 x}-u u_{x x} e^{-2 x}\right) \\
& \left.+c_{3}\left(2 u u_{x} e^{2 x}-u_{x}^{2} e^{2 x}-u u_{x x} e^{2 x}\right)\right]=0 .
\end{aligned}
$$

Therefore the conserved vectors for the Degasperis Procesi equation (7) are

$$
\begin{gathered}
T_{1}^{1}=u-u_{x x}, \quad T_{1}^{2}=2 u^{2}-u_{x}^{2}-u u_{x x}, \\
T_{2}^{1}=u e^{-2 x}-u_{x x} e^{-2 x} \\
T_{2}^{2}=-2 u u_{x} e^{-2 x}-u_{x}^{2} e^{-2 x}-u u_{x x} e^{-2 x} \\
T_{3}^{1}=u e^{2 x}-u_{x x} e^{2 x} \\
T_{3}^{2}=2 u u_{x} e^{2 x}-u_{x}^{2} e^{2 x}-u u_{x x} e^{2 x}
\end{gathered}
$$

The variational derivative approach for the Degasperis Procesi equation gives three multipliers of the form $A(t, x, u)$ and hence three conserved vectors are obtained.

\section{Conservation Laws for a Coupled Variable-Coefficient Modified Korteweg-de Vries System in a Two-Layer Fluid Model}

In this section we recall some basic definitions related to the multiplier approach.

Let $(x, t)$ be two independent variables and let $(u, v)$ be dependent variables.

(1) The total derivative operators $D_{t}$ and $D_{x}$ are

$$
\begin{aligned}
D_{t}= & \frac{\partial}{\partial t}+u_{t} \frac{\partial}{\partial u}+v_{t} \frac{\partial}{\partial v}+u_{t t} \frac{\partial}{\partial u_{t}}+v_{t t} \frac{\partial}{\partial v_{t}} \\
& +u_{t x} \frac{\partial}{\partial u_{x}}+v_{t x} \frac{\partial}{\partial v_{x}}+\cdots, \\
D_{x}= & \frac{\partial}{\partial x}+u_{x} \frac{\partial}{\partial u}+v_{x} \frac{\partial}{\partial v}+u_{x x} \frac{\partial}{\partial u_{x}} \\
& +v_{x x} \frac{\partial}{\partial v_{x}}+u_{t x} \frac{\partial}{\partial u_{t}}+v_{t x} \frac{\partial}{\partial v_{t}}+\cdots .
\end{aligned}
$$

(2) The standard Euler operators $\delta / \delta u$ and $\delta / \delta v$ are

$$
\begin{gathered}
\frac{\delta}{\delta u}=\frac{\partial}{\partial u}-D_{t} \frac{\partial}{\partial u_{t}}-D_{x} \frac{\partial}{\partial u_{x}}+D_{t}^{2} \frac{\partial}{\partial u_{t t}} \\
+D_{x}^{2} \frac{\partial}{\partial u_{x x}}+D_{x} D_{t} \frac{\partial}{\partial u_{t x}}-\cdots, \\
\frac{\delta}{\delta v}=\frac{\partial}{\partial v}-D_{t} \frac{\partial}{\partial v_{t}}-D_{x} \frac{\partial}{\partial v_{x}}+D_{t}^{2} \frac{\partial}{\partial v_{t t}} \\
+D_{x}^{2} \frac{\partial}{\partial v_{x x}}+D_{x} D_{t} \frac{\partial}{\partial v_{t x}}-\cdots
\end{gathered}
$$

Consider a $k$ th-order system of two partial differential equations of two independent and two dependent variables

$$
\begin{aligned}
& E_{1}\left(t, x, u, v, u_{t}, v_{t}, \ldots\right), \\
& E_{2}\left(t, x, u, v, u_{t}, v_{t}, \ldots\right) .
\end{aligned}
$$

(3) A vector $T=\left(T^{1}, T^{2}\right)$ satisfying

$$
D_{t} T^{1}+D_{x} T^{2}=0
$$

for all solutions of (19) is known as the conserved vector of (19).

(4) The multipliers $A_{1}, A_{2}$ of system (19) have the property

$$
D_{t} T^{1}+D_{x} T^{2}=A_{1} E_{1}+A_{2} E_{2}
$$

for the arbitrary functions $u(x, t), v(x, t)$.

(5) The determining equations for the multipliers are obtained by taking variational derivative of (21):

$$
\begin{aligned}
& \frac{\delta}{\delta u}\left[A_{1} E_{1}+A_{2} E_{2}\right]=0, \\
& \frac{\delta}{\delta v}\left[A_{1} E_{1}+A_{2} E_{2}\right]=0 .
\end{aligned}
$$

Equation (22) holds for the arbitrary functions $u(x, t)$, $v(x, t)$ not only for the solutions of system (19). Equation (22) yields multipliers for all local conservation laws. Then conserved vectors can be derived systematically using (21) as the determining equation. But in some problems it is not difficult to construct the conserved vectors by elementary manipulations once the multiplier has been determined.

Example 1. Consider a coupled variable-coefficient modified Korteweg-de Vries system in a two-layer fluid model

$$
\begin{aligned}
& u_{t}-\alpha(t)\left[u_{x x x}+6\left(u^{2}-v^{2}\right) u_{x}-12 u v v_{x}\right]-4 \beta(t) u_{x}=0, \\
& v_{t}-\alpha(t)\left[v_{x x x}+6\left(u^{2}-v^{2}\right) v_{x}+12 u v u_{x}\right]-4 \beta(t) v_{x}=0 .
\end{aligned}
$$

System (23) was proposed in [18] as an important particular case of the formidable generalized coupled variablecoefficient modified Korteweg-de Vries (CVmKdV) system. The $(\mathrm{CVmKdV})$ system was derived by Gao and Tang [19] as a two-layer model describing atmospheric and oceanic phenomena like interactions between the atmosphere and ocean, atmospheric blocking, oceanic circulations, hurricanes, typhoons, and so forth. 
The determining equations for multipliers of the forms $A_{1}(x, t, u, v)$ and $A_{2}(x, t, u, v)$ from (22) are

$$
\begin{gathered}
\frac{\delta}{\delta u}\left[A _ { 1 } \left(u_{t}-\alpha(t)\left[u_{x x x}+6\left(u^{2}-v^{2}\right) u_{x}-12 u v v_{x}\right]\right.\right. \\
\left.-4 \beta(t) u_{x}\right) \\
+A_{2}\left(v_{t}-\alpha(t)\left[v_{x x x}+6\left(u^{2}-v^{2}\right) v_{x}+12 u v u_{x}\right]\right. \\
\left.\left.-4 \beta(t) v_{x}\right)\right]=0 \\
\frac{\delta}{\delta v}\left[A _ { 1 } \left(u_{t}-\alpha(t)\left[u_{x x x}+6\left(u^{2}-v^{2}\right) u_{x}-12 u v v_{x}\right]\right.\right. \\
\left.-4 \beta(t) u_{x}\right) \\
+A_{2}\left(v_{t}-\alpha(t)\left[v_{x x x}+6\left(u^{2}-v^{2}\right) v_{x}+12 u v u_{x}\right]\right. \\
\left.\left.-4 \beta(t) v_{x}\right)\right]=0
\end{gathered}
$$

where the standard Euler operators $\delta / \delta u$ and $\delta / \delta v$ are defined in (17) and (18), respectively. Expansion of (24) yields

$$
\begin{aligned}
& A_{1 u}\left[u_{t}-\alpha(t)\left[u_{x x x}+6\left(u^{2}-v^{2}\right) u_{x}-12 u v v_{x}\right]\right. \\
& \left.-4 \beta(t) u_{x}\right] \\
& +A_{2 u}\left[v_{t}-\alpha(t)\left[v_{x x x}+6\left(u^{2}-v^{2}\right) v_{x}+12 u v u_{x}\right]\right. \\
& \left.-4 \beta(t) v_{x}\right] \\
& +\left(12 \alpha v v_{x}-12 \alpha u u_{x}\right) A_{1}-\left(12 \alpha u v_{x}+12 \alpha v u_{x}\right) A_{2} \\
& -D_{t}\left(A_{1}\right)+D_{x}\left[\left(6 \alpha\left(u^{2}-v^{2}\right)+4 \beta\right) A_{1}+12 \alpha u v A_{2}\right] \\
& +D_{x}^{3}\left(\alpha A_{1}\right) \\
& A_{1 v}\left[u_{t}-\alpha(t)\left[u_{x x x}+6\left(u^{2}-v^{2}\right) u_{x}-12 u v v_{x}\right]\right. \\
& \left.-4 \beta(t) u_{x}\right] \\
& +A_{2 v}\left[v_{t}-\alpha(t)\left[v_{x x x}+6\left(u^{2}-v^{2}\right) v_{x}+12 u v u_{x}\right]\right. \\
& \left.-4 \beta(t) v_{x}\right] \\
& +\left(12 \alpha v v_{x}-12 \alpha u u_{x}\right) A_{2}+\left(12 \alpha u v_{x}+12 \alpha v u_{x}\right) A_{1} \\
& -D_{t}\left(A_{2}\right)+D_{x}\left[\left(6 \alpha\left(u^{2}-v^{2}\right)+4 \beta\right) A_{2}-12 \alpha u v A_{1}\right] \\
& +D_{x}^{3}\left(\alpha A_{2}\right) \text {. }
\end{aligned}
$$

Equations (25) and (26) are separated according to different combinations of derivatives of $u$ and $v$ and, after some simplification following system of equations for $A_{1}, A_{2}$ is obtained:

$$
\begin{gathered}
A_{1 u u}=0, \quad A_{1 v v}=0, \quad A_{1 u x}=0, \\
A_{1 v x}=0, \quad A_{1 u v}=0, \\
A_{1 v}-A_{2 u}=0, \quad A_{1 u}+A_{2 v}=0, \\
\left(6 \alpha\left(u^{2}-v^{2}\right)+4 \beta\right) A_{1 x} \\
+12 \alpha u v A_{2 x}-A_{1 t}+\alpha A_{1 x x x}=0, \\
A_{2 u u}=0, \quad A_{2 v v}=0, \quad A_{2 u x}=0, \\
A_{2 v x}=0, \quad A_{2 u v}=0, \\
\left(6 \alpha\left(u^{2}-v^{2}\right)+4 \beta\right) A_{2 x} \\
-12 \alpha u v A_{1 x}-A_{2 t}+\alpha A_{2 x x x}=0 .
\end{gathered}
$$

Solution of system (27) yields

$$
\begin{aligned}
& A_{1}=c_{1}+c_{3} u+c_{4} v, \\
& A_{2}=c_{2}-c_{3} v+c_{4} u,
\end{aligned}
$$

where $c_{1}, c_{2}, c_{3}$, and $c_{4}$ are constants.

Equations (21) and (28) give the following conserved vectors satisfying (20):

$$
\begin{gathered}
T_{1}^{1}=u, \quad T_{1}^{2}=-2 \alpha u^{3}-\alpha u_{x x}+6 \alpha u v^{2}-4 \beta u, \\
T_{2}^{1}=v, \quad T_{2}^{2}=2 \alpha v^{3}-\alpha v_{x x}-6 \alpha u^{2} v-4 \beta v, \\
T_{3}^{1}=\frac{u^{2}}{2}-\frac{v^{2}}{2}, \\
T_{3}^{2}=\alpha\left(v v_{x x}-u u_{x x}\right)+9 \alpha u^{2} v^{2}+\alpha\left(\frac{u_{x}^{2}}{2}-\frac{v_{x}^{2}}{2}\right) \\
+v^{2}\left(2 \beta-\frac{3}{2} \alpha v^{2}\right)-u^{2}\left(\frac{3}{2} \alpha u^{2}+2 \beta\right), \\
T_{4}^{1}=u v, \\
T_{4}^{2}=-6 \alpha u^{3} v+\alpha\left(u_{x} v_{x}-v u_{x x}\right) \\
+u\left(6 \alpha v^{3}-4 v \beta-\alpha v_{x x}\right) .
\end{gathered}
$$

\section{Conclusions}

The conservation laws for the Degasperis Procesi equation and a coupled variable-coefficient modified Korteweg-de Vries system in a two-layer fluid model were established with the help of the multiplier approach. The multiplier approach on the Degasperis Procesi equation yielded three multipliers and thus three local conserved vectors were obtained in each case. The multiplier approach when applied to a coupled variable-coefficient modified Korteweg-de Vries system in a two-layer fluid model gave four multipliers of form $A(x, t, u, v)$. Each multiplier corresponds to a conserved vector and thus four local conserved vectors were obtained. 


\section{Conflict of Interests}

The authors declare that there is no conflict of interests regarding the publication of this paper.

\section{References}

[1] P. S. Laplace, Traité de Mécanique Céleste, vol. 1, Paris, 1798. English Translation, Celestrial Mechanics, New York, NY, USA, 1966.

[2] P. J. Olver, Applications of Lie Groups to Differential Equations, Springer, New York, NY, USA, 1993.

[3] S. C. Anco and G. Bluman, "Direct construction method for conservation laws of partial differential equations. Part I: examples of conservation law classifications," European Journal of Applied Mathematics, vol. 13, no. 5, pp. 545-566, 2002.

[4] A. H. Kara and F. M. Mahomed, "Relationship between symmetries and conservation laws," International Journal of Theoretical Physics, vol. 39, no. 1, pp. 23-40, 2000.

[5] T. Wolf, "A comparison of four approaches to the calculation of conservation laws," European Journal of Applied Mathematics, vol. 13, no. 2, pp. 129-152, 2002.

[6] T. Wolf, A. Brand, and M. Mohammadzadeh, "Computer algebra algorithms and routines for the computation of conservation laws and fixing of gauge in differential expressions," Journal of Symbolic Computation, vol. 27, no. 2, pp. 221-238, 1999.

[7] Ü. Göktaş and W. Hereman, "Symbolic computation of conserved densities for systems of nonlinear evolution equations," Journal of Symbolic Computation, vol. 24, no. 5, pp. 591-621, 1997.

[8] W. Hereman, P. J. Adams, H. L. Eklund, M. S. Hickman, and B. M. Herbst, "Direct methods and symbolic software for conservation laws of nonlinear equations," in Advances of Nonlinear Waves and Symbolic Computation, Z. Yan, Ed., chapter 2, pp. 1979, Nova Science Publishers, New York, NY, USA, 2009.

[9] W. Hereman, M. Colagrosso, R. Sayers et al., "Continuous and discrete homotopy operators and the computation of conservation laws," in Differential Equations with Symbolic Computation, D. Wang and Z. Zheng, Eds., pp. 249-285, Birkhäuser, 2005.

[10] W. Hereman, "Symbolic computation of conservation laws of nonlinear partial differential equations in multi-dimensions," International Journal of Quantum Chemistry, vol. 106, no. 1, pp. 278-299, 2006.

[11] A. F. Cheviakov, "GeM software package for computation of symmetries and conservation laws of differential equations," Computer Physics Communications, vol. 176, no. 1, pp. 48-61, 2007.

[12] Y.-F. Wang, B. Tian, M. Li, P. Wang, and M. Wang, "Integrability and soliton-like solutions for the coupled higher-order nonlinear Schrödinger equations with variable coefficients in inhomogeneous optical fibers," Communications in Nonlinear Science and Numerical Simulation, vol. 19, no. 6, pp. 1783-1791, 2014.

[13] Z. Hui-Ling, T. Bo, W. Yu-Feng, Z. H. Hui, and S. Wen-Rong, "Dynamic behavior of the quantum Zakharov-Kuznetsov equations in dense quantum magnetoplasmas," Physics of Plasmas, vol. 21, Article ID 012304, 2014.

[14] Y.-J. Shen, Y.-T. Gao, D.-W. Zuo, Y.-H. Sun, Y.-J. Feng, and L. Xue, "Nonautonomous matter waves in a spin-1 Bose-Einstein condensate," Physical Review E, vol. 89, no. 6, Article ID 062915, 12 pages, 2014.
[15] H. Steudel, "Über die Zuordnung zwischen Invarianzeigenschaften und Erhaltungssatzen," Zeitschrift für Naturforschung, vol. 17A, pp. 129-132, 1962.

[16] P. J. Olver, Applications of Lie Groups to Differential Equations, Springer, New York, NY, USA, 1993.

[17] A. Degasperis and M. Procesi, "Asymptotic integrability", in Symmetry and Perturbation Theory, A. Degasperis and G. Gaeta, Eds., pp. 23-27, World Scientific Publishers, Singapore, 1999.

[18] S.-H. Zhu, Y.-T. Gao, X. Yu, Z.-Y. Sun, X.-L. Gai, and D.-X. Meng, "Painlevé property, soliton-like solutions and complexitons for a coupled variable-coefficient modified Korteweg-de Vries system in a two-layer fluid model," Applied Mathematics and Computation, vol. 217, no. 1, pp. 295-307, 2010.

[19] Y. Gao and X.-Y. Tang, "A coupled variable coefficient modified $\mathrm{KdV}$ equation arising from a two-layer fluid system," Communications in Theoretical Physics, vol. 48, no. 6, pp. 961-970, 2007. 


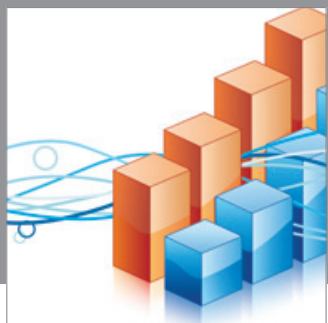

Advances in

Operations Research

mansans

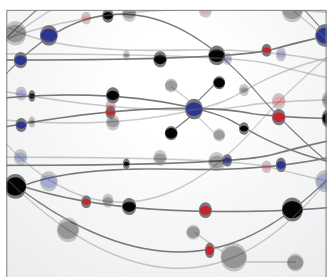

The Scientific World Journal
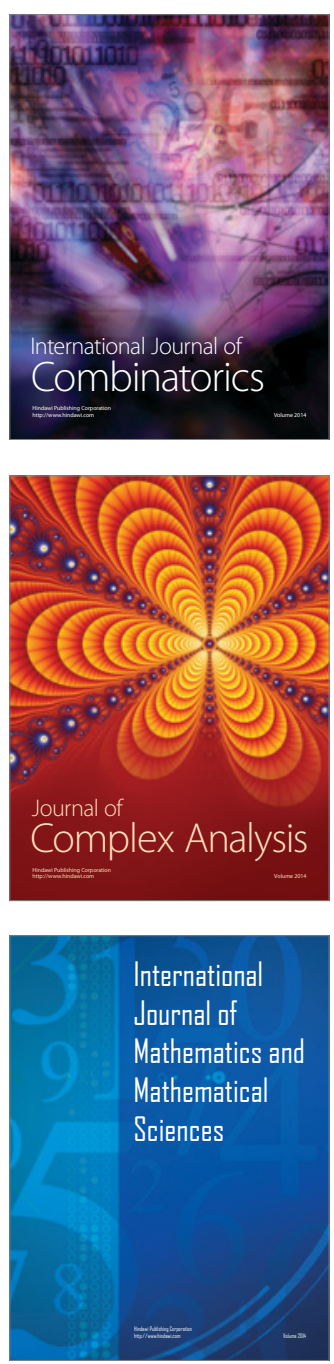
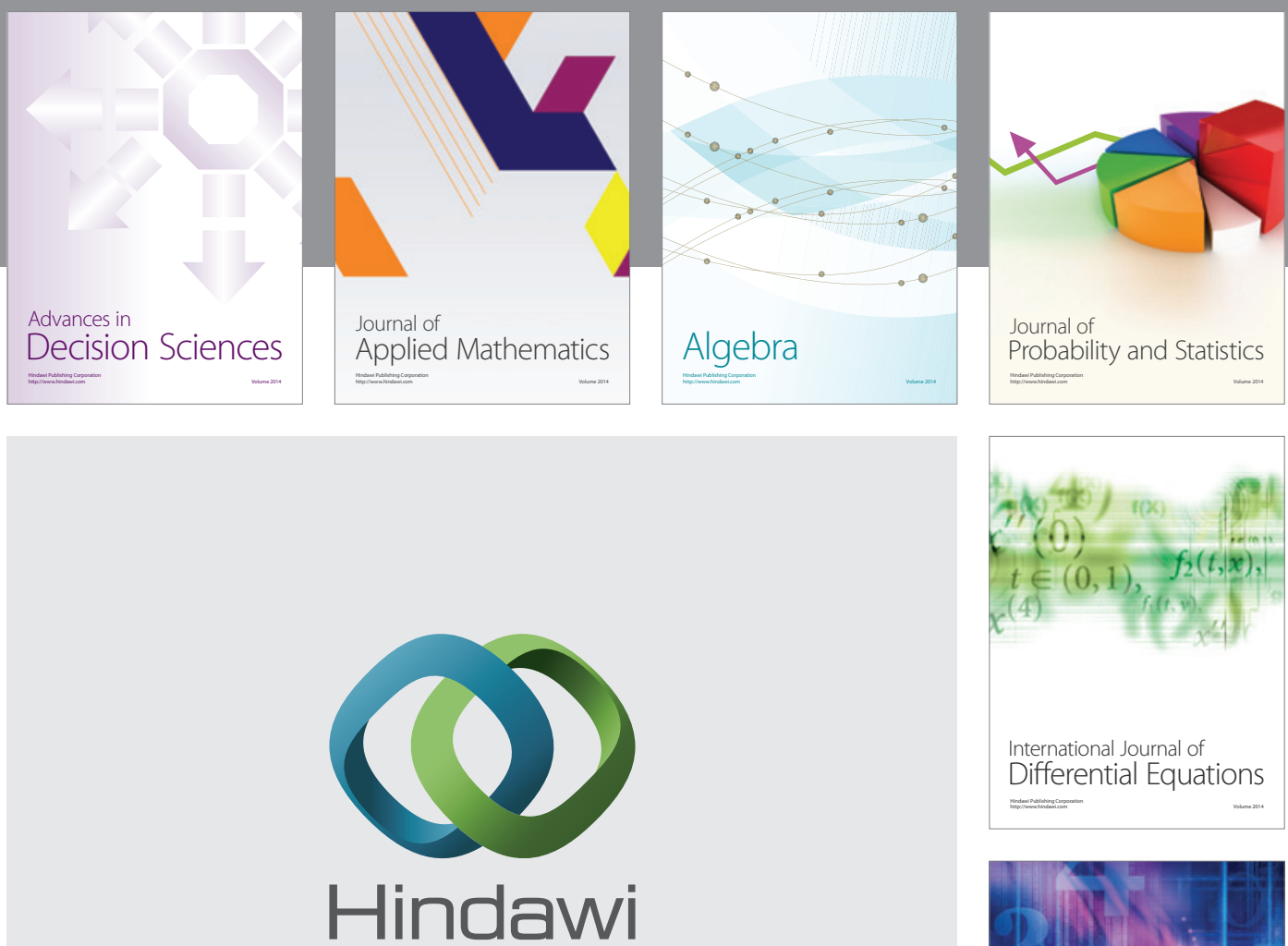

Submit your manuscripts at http://www.hindawi.com
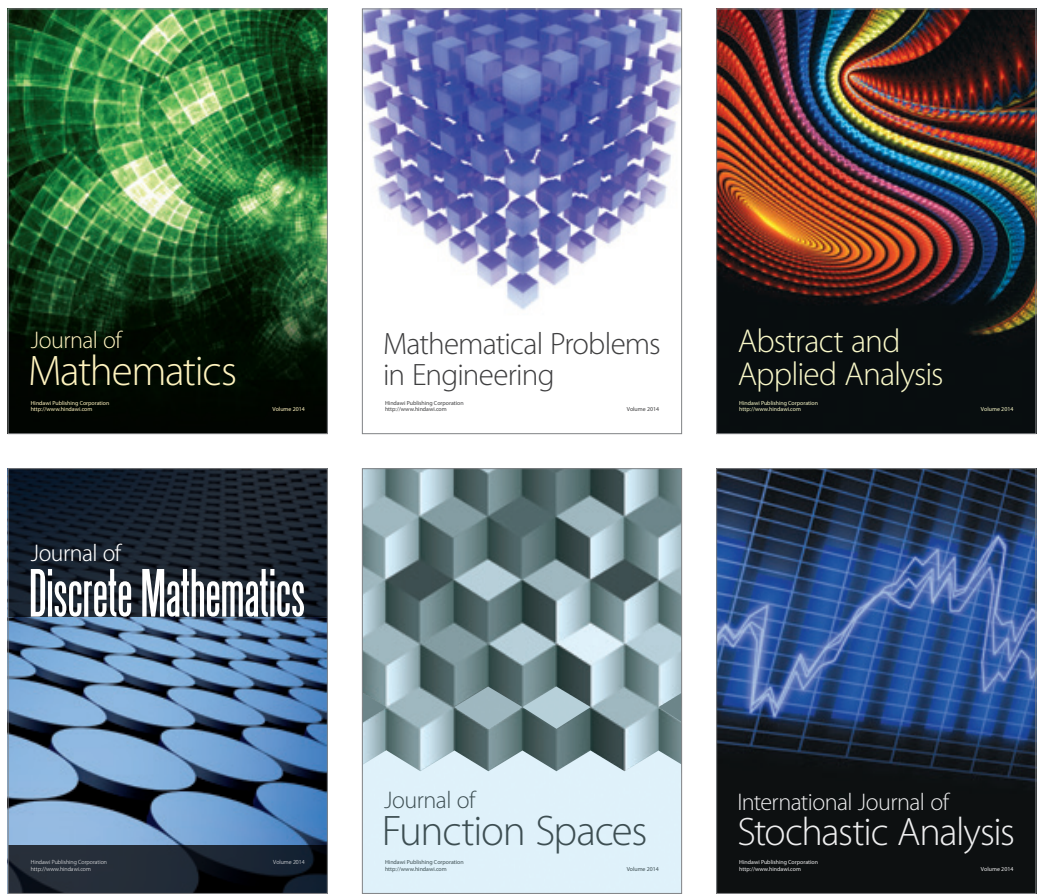

Journal of

Function Spaces

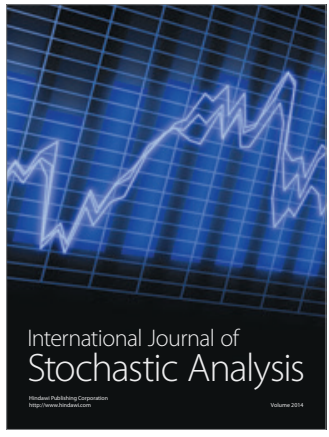

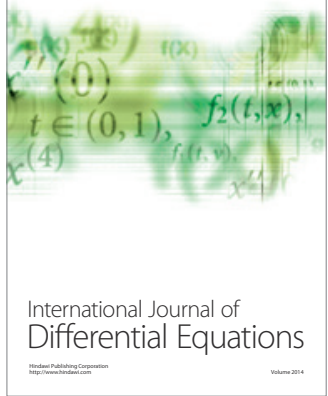
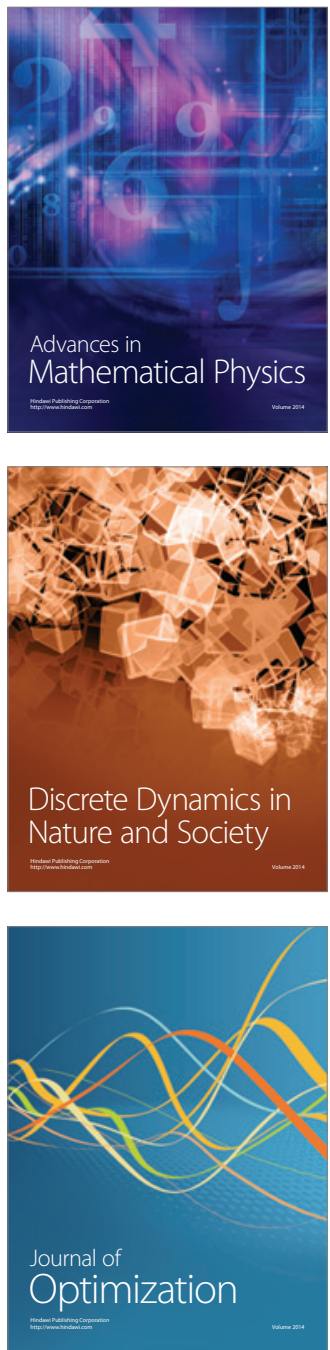